\title{
PREDIÇÃO DAS PROPRIEDADES FÍSICAS E TERMODINÂMICAS DO ÓLEO DA POLPA DA MACAÚBA E BIODIESEL POR MÉTODO DE CONTRIBUIÇÃO DE GRUPOS
}

\author{
G. C. RABELO SILVA ${ }^{1}$ e M.H. CANO ANDRADE ${ }^{2}$ \\ ${ }^{1}$ Universidade Federal de São João Del Rei, Curso de Bioquímica \\ ${ }^{2}$ Universidade Federal de Minas Gerais, Departamento de Engenharia Química \\ E-mail para contato: giselec@ufsj.edu.br
}

\begin{abstract}
RESUMO - O conhecimento das propriedades físicas e termodinâmicas dos óleos em processos oleoquímicos, principalmente envolvidos na produção de biodiesel são essenciais para a engenharia de processo. Apesar de vários estudos relatarem essas propriedades para alguns compostos, há ainda uma necessidade de expandir o banco de dados existentes na literatura. Em contraste com os métodos tradicionais baseados em correlações de dependência da temperatura, neste trabalho, os métodos de contribuição de grupo foram aplicados, não só pela sua aproximação com os valores reais como também pela facilidade na sua implementação. O objetivo do trabalho foi utilizar alguns métodos de estimativa para predizer algumas propriedades do óleo da polpa da macaúba e do biodiesel, a partir da composição inicial dos ácidos graxos, a saber: temperatura de ebulição; temperatura, pressão e volume críticos; energia livre de Gibbs de formação padrão e entalpia de formação. As estimativas foram comparadas com dados da literatura. Os resultados apresentaram alguns desvios entre as estimativas e os valores encontrados na literatura e mostraram a importância da comparação entre os diferentes métodos de estimativas para a predição de uma única propriedade, principalmente no caso de moléculas complexas.
\end{abstract}

\section{INTRODUÇÃO}

A utilização de biocombustíveis como substitutos dos combustíveis derivados do petróleo, tem sido realizada para amenizar problemas, tais como agravamento do efeito estufa, poluição ambiental e instabilidade econômica. Sendo assim, o biodiesel tem atraído a atenção de muitas pesquisas com o objetivo de viabilizar e otimizar sua produção.

O biodiesel pode ser definido como uma mistura de monoesteres metílicos ou etílicos de cadeia longa proveniente de matérias-primas renováveis. Demirbas (2005) define que o biodiesel é uma variedade de combustível oxigenado a base de ésteres derivados de fontes renováveis. $\mathrm{O}$ biodiesel pode ser obtido por reações de esterificação das referidas matérias-primas; ou reações de transesterificação. Nestas reações, a matéria-prima reage com um álcool de cadeia curta (metanol ou etanol) resultando no monoester (biodiesel) e no glicerol (glicerina), na presença de um catalisador. 
O custo da produção do biodiesel, atualmente está vinculado ao valor da matéria-prima. Por isso, existe uma demanda por novas oleaginosas de alto potencial e elevada produtividade. A Macaúba (Acrocomia aculeata) é uma palmeira, típica do cerrado brasileiro, que produz um fruto capaz de ser utilizado como fonte promissora de óleo vegetal. Diversos estudos têm sido realizados com esse fruto para avaliar a produção de biodiesel (Rezende, 2011; Lopes et al. 2013; Basso et al, 2013).

Para a otimização do processo de produção do biodiesel, dados das propriedades físicas e termodinâmicas são de fundamental importância. Algumas destas propriedades possuem medição experimental complexa e até mesmo impossível, devido à degradação do composto, sendo então a literatura limitada em dados termodinâmicos tabelados, para os ácidos graxos e ésteres.

Devido a grande variedade de espécies de ésteres e, em particular, os triglicerídeos nos óleos vegetais, a metodologia de contribuição de grupos e equações de estado é utilizado para a estimativa das propriedades de componentes puros. Considerando que os métodos de contribuição de grupo geralmente requerem apenas o conhecimento sobre a estrutura molecular, os métodos correspondentes de estado são baseados num número muito limitado de valores de propriedades, tais como o ponto crítico e um valor de pressão de vapor na proximidade do ponto de ebulição normal e, portanto, não são verdadeiramente preditivos.

Assim, estudos de desenvolvimento de métodos de contribuição de grupos têm sido reportados para os produtos e matéria-prima no processo de produção do biodiesel. Zeberg e Stenby (1999) desenvolveram, um modelo de grupo de contribuição para a previsão de pontos de fusão e entalpias de fusão de triglicerídeos. Ceriani et al. (2003) apresentaram um modelo de contribuição de grupo para estimar pressões de vapor e calores de vaporização de compostos graxos.

Neste trabalho, por comparação com dados experimentais disponíveis na literatura, Banco de Dados Dortmund (DDB, 2014) e National Institute of Standards and technology (NIST, 2014), verificou-se a aplicabilidade dos modelos estabelecidos para compostos orgânicos, os óleos vegetais e biodiesel. $\mathrm{O}$ foco foi a estimativa da temperatura de ebulição, temperatura, pressão e volume críticos, energia livre de Gibbs de formação padrão e entalpia de formação, a partir da composição inicial dos ácidos graxos do óleo da macaúba e seu respectivo biodiesel, por metanol ou etanol.

\section{METODOLOGIA}

Todos os métodos apresentados a seguir foram implementados no simulador Aspen Plus® versão 8.0. Para o uso de métodos de contribuição de grupos, as moléculas foram estudadas, os grupos funcionais foram identificados e quantificados.

\subsection{Temperatura Normal de Ebulição $\left(T_{b}\right)$}

Os pontos de ebulição normal foram estimados pelo método de Constantinou e Gani (1994) e, Joback e Reid (1987), doravante denominados GANI e JOBACK, respectivamente. As estimativas por estes métodos são exclusivamente via contribuição de grupo, não necessitando de quaisquer outras informações sobre a molécula.

O método JOBACK divide os grupos de sua estimativa em acíclicos, cíclicos e oxigenados. 
O método GANI divide seu método de estimativa em dois níveis: grupos de primeira e grupos de segunda ordem.

Para escolher o método mais preciso, os valores das propriedades estimados foram comparados com valores experimentais relatados na literatura.

\subsection{Propriedades Críticas $\left(T_{c}, P_{c}, V_{c}\right)$}

Para as propriedades críticas, pressão $(\mathrm{Pc})$, volume $(\mathrm{Vc})$ e temperatura $(\mathrm{Tc})$, os métodos utilizados foram: GANI, JOBACK, Ambrose (Reid et al.,1987) e Lydersen (Reid et al.,1987). O primeiro método necessita apenas da informação da estrutura do composto, enquanto que os demais, além de serem baseados na estrutura molecular do composto, necessitam dos valores da massa molar e temperatura de ebulição. Os valores dessa última propriedade foram obtidos da estimativa do método de GANI, conforme demonstrado no item anterior.

\subsection{Energia livre de Gibbs e Entalpia de Formação}

Para a entalpia de formação os métodos utilizados foram: GANI, JOBACK e Benson (Reid et al.,1987).

\subsection{Propriedades do Óleo da Polpa da Macaúba}

As estimativas das propriedades neste trabalho foram feitas para os ácidos graxos do óleo da polpa da macaúba e para o biodiesel de tal óleo, considerando uma reação de transesterificação com rendimento igual a $100 \%$, seja com metanol ou etanol.

Assim, adotou-se que a porcentagem de ácidos graxos do óleo da macaúba (encontrada na literatura) é similar a porcentagem dos ésteres metílicos (etílicos) de cada ácido no biocombustível. Segundo Pimenta (2010), a composição do óleo da macaúba em ácido graxo é: 0,03\% de ácido mirístico (C14:0), 16,51\% palmítico (C16:0), 2,92\% palmitoleico (C16:1), 2,89\% esteárico (C18:0), 67,67\% oleico (C18:1), 8,82\% linoleico (C18:2), 0,81\% linolênico (C18:3) e 0,16\% araquídico (C20:0). As propriedades dos óleos e dos biodieseis foram obtidas pela média ponderada das propriedades dos ácidos e dos ésteres, considerando as porcentagens apresentadas. Neste trabalho foram levados em consideração os ácidos de cadeia carbônica entre C14 a C20. Os demais ácidos apresentam-se em quantidades desprezíveis na composição do óleo.

\section{RESULTADOS E DISCUSSÃO}

\subsection{Temperatura Normal de Ebulição}

Nas Figuras 1(a) e 1(b) podem ser observadas as temperaturas de ebulição estimadas dos ácidos graxos e ésteres metílicos, respectivamente.

Nestas figuras, os pontos de ebulição dos ácidos graxos saturados mostram uma característica ligeiramente curva, quase linear, como Cordes (2002) demonstrou em séries análogas. A curva de tendência dos ácidos graxos saturados apresentam para JOBACK $\mathrm{r}^{2}=0,9942$ e para GANI 0,9981, no entanto, para os ésteres metílicos de cadeia saturada observou-se para o método de JOBACK $\mathrm{r}^{2}=1$ e para GANI $\mathrm{r}^{2}=0,9979$. Adicionalmente, observa-se que a temperatura de ebulição diminui com o aumento do grau de insaturação (número de ligações duplas $\mathrm{C}-\mathrm{C}$ ). 


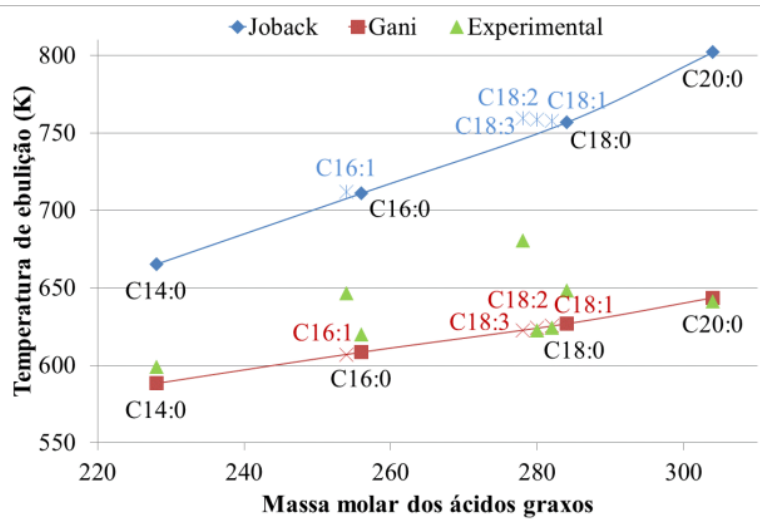

(a)

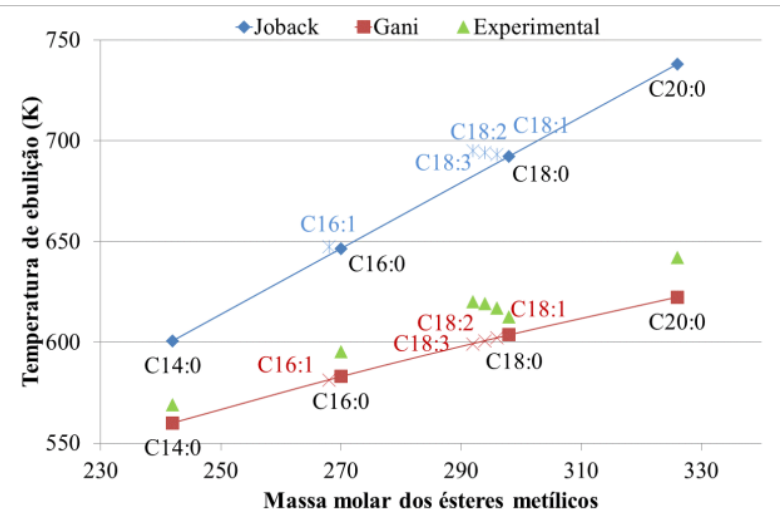

(b)

Figura 1- Temperaturas de ebulição estimada em função da massa molar. (a) Ácidos graxos. (b) ésteres metílicos.

A comparação das temperaturas obtidas em cada modelo foi realizada através dos desvios,

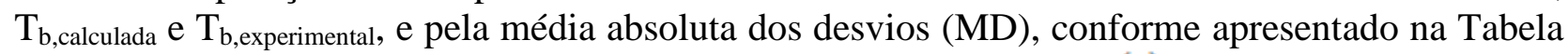
1. A média dos desvios foi calculada de acordo com a equação $M D=\left(\frac{1}{n}\right) \sum_{i=1}^{n}\left|x_{i_{i} c a l c}-x_{i_{i} \exp }\right|$.

As Figuras 1(a) e 1(b), e a Tabela 1 mostram que os métodos de GANI e JOBACK apresentam boa concordância de comportamento em relação à variação na massa molar dos ácidos graxos e dos ésteres metílicos. Contudo, entre os dois métodos, GANI foi o que apresentou os melhores valores de estimativa da temperatura de ebulição, tanto dos ésteres quanto dos ácidos. Vale observar que o método JOBACK, em geral, superestima a propriedade estudada.

Tabela 1 - Dados experimentais, desvios e média absoluta dos desvios para as temperaturas de ebulição estimadas dos ácidos graxos e ésteres metílicos

\begin{tabular}{|c|c|c|c|c|c|c|}
\hline \multicolumn{4}{|c|}{ Ácidos graxos } & \multicolumn{3}{|c|}{ Ésteres metílicos } \\
\hline & \multirow{2}{*}{$T_{b, \exp } 1(K)$} & \multicolumn{2}{|c|}{$\mathbf{T}_{\mathbf{b}, \text { calc }}-\mathbf{T}_{\mathbf{b}, \mathbf{e x p}}$} & \multirow{2}{*}{$T_{b, \exp } 1(K)$} & \multicolumn{2}{|c|}{$\mathbf{T}_{\mathbf{b , c a l c}}-\mathbf{T}_{\mathbf{b , e x p}}$} \\
\hline & & Joback & Gani & & Joback & Gani \\
\hline C14:0 & 599,00 & 66,23 & $-10,69$ & 568,90 & 31,92 & $-8,87$ \\
\hline C16:0 & 620,10 & 90,89 & $-11,63$ & 595,20 & 51,38 & $-12,18$ \\
\hline C16:1 & $648,20^{2}$ & 63,73 & $-41,21$ & - & - & - \\
\hline C18:0 & 641,30 & 115,45 & $-14,48$ & 612,70 & 79,64 & $-9,01$ \\
\hline C18:1 & 646,57 & 111,12 & $-21,11$ & 617,00 & 76,28 & $-14,84$ \\
\hline C18:2 & 624,10 & 134,53 & $-0,002$ & 619,20 & 75,02 & $-18,57$ \\
\hline C18:3 & 622,70 & 136,87 & 0,02 & 620,20 & 74,96 & $-21,11$ \\
\hline \multirow[t]{2}{*}{ C20:0 } & 680,60 & 121,50 & $-36,94$ & 642,20 & 95,90 & $-19,75$ \\
\hline & $M D$ & 105,040 & $-17,004$ & $M D$ & 60,637 & 13,041 \\
\hline
\end{tabular}

${ }^{1}$ Nist- National Institute of Standards and Technology. ${ }^{2}$ DDB- Dortmund Data Bank.

Desde que dados experimentais da temperatura de ebulição de ésteres etílicos não estão disponíveis na literatura, neste trabalho, o método GANI foi utilizado para estimar esses dados, conforme apresentado na Tabela 2. 
Tabela 2 - Temperaturas de ebulição estimadas para os ésteres etílicos, utilizando o método de Gani e Constantinou (1994)

\begin{tabular}{cccccc}
\hline $\begin{array}{c}\text { Ésteres Etílicos } \\
\text { saturados }\end{array}$ & $\begin{array}{c}\text { Massa } \\
\text { molar }\end{array}$ & $\begin{array}{c}\mathbf{T}_{\text {b,calc }} \\
(\mathbf{K})\end{array}$ & $\begin{array}{c}\text { Ésteres Etílicos } \\
\text { Insaturados }\end{array}$ & $\begin{array}{c}\text { Massa } \\
\text { molar }\end{array}$ & $\begin{array}{c}\mathbf{T}_{\mathbf{b}, \text { calc }} \\
(\mathbf{K})\end{array}$ \\
\hline C14:0 & 256 & 571,85 & $\mathbf{C 1 6 : 1}$ & 282 & 592,02 \\
C16:0 & 284 & 593,61 & $\mathbf{C 1 8 : 1}$ & 310 & 611,83 \\
C18:0 & 321 & 613,28 & $\mathbf{C 1 8 : 2}$ & 308 & 610,37 \\
C20:0 & 340 & 631,23 & $\mathbf{C 1 8 : 3}$ & 306 & 608,90 \\
\hline
\end{tabular}

Destaca-se que o método GANI também foi escolhido para a predição das temperaturas de ebulição dos óleos vegetais e seus respectivos ésteres metílicos e etílicos.

\subsection{Propriedades Críticas}

Os dados estimados para a temperatura crítica podem ser observados na Tabela 3. Os valores foram comparados através dos desvios, calculado pela equação $\left[\mathrm{T}_{\mathrm{c}, \text { calculada }}-\mathrm{T}_{\mathrm{c}, \text { experimental }}\right]$, assim como a média absoluta dos desvios (MD).

Tabela 3 - Dados experimentais, desvios e média absoluta dos desvios para as temperaturas de críticas estimadas dos ácidos graxos e ésteres metílicos

\begin{tabular}{|c|c|c|c|c|c|}
\hline & \multirow{2}{*}{$T_{c, \exp }{ }^{1}(K)$} & \multicolumn{4}{|c|}{$\mathbf{T}_{c, \text { calc }}-\mathbf{T}_{\mathbf{c}, \exp }$} \\
\hline & & Joback & Gani & Lydersen & Ambrose \\
\hline \multicolumn{6}{|c|}{ Ácidos graxos } \\
\hline C16:0 & 785 & $-26,19$ & $-4,62$ & $-28,57$ & $-20,70$ \\
\hline C18:0 & 803 & $-33,16$ & $-6,35$ & $-35,17$ & $-30,46$ \\
\hline C18:1 & 796 & $-44,49$ & 0,34 & $-46,41$ & $-36,41$ \\
\hline C18:2 & 787 & $-9,10$ & 9,03 & $-10,99$ & 5,44 \\
\hline C18:3 & 796 & $-18,80$ & $-0,28$ & $-20,54$ & 2,51 \\
\hline MD dos ác & & $-16,467$ & $-0,234$ & $-17,709$ & $-9,952$ \\
\hline \multicolumn{6}{|c|}{ Ésteres metílicos } \\
\hline C16:0 & 792 & $-60,28$ & $-37,71$ & $-62,62$ & $-54,77$ \\
\hline C18:0 & 804 & $-55,89$ & $-31,05$ & $-58,04$ & $-52,33$ \\
\hline C18:1 & 768 & 25,34 & 4,60 & 23,18 & 34,94 \\
\hline MD dos ést & metílicos & $-11,354$ & $-8,021$ & $-12,185$ & $-9,019$ \\
\hline \multicolumn{6}{|c|}{ Ésteres etílicos } \\
\hline C16:0 & 803 & $-63,37$ & $-39,14$ & $-65,65$ & $-58,65$ \\
\hline C16:1 & 796 & $-43,12$ & $-32,51$ & $-45,26$ & $-32,61$ \\
\hline C18:0 & 820 & $-62,83$ & $-38,40$ & $-64,77$ & $-60,84$ \\
\hline \multicolumn{2}{|c|}{ MD dos ésteres etílicos } & $-21,165$ & $-13,757$ & $-21,959$ & $-19,011$ \\
\hline
\end{tabular}

Nota-se, a partir da análise da Tabela 3, que o método de GANI apresentou os menores desvios e média absoluta dos desvios, tanto para os ácidos graxos como para os ésteres. Portanto, esse método foi escolhido para a predição das temperaturas críticas dos óleos vegetais e dos respectivos ésteres metílicos e etílicos. 
Os dados obtidos para a pressão crítica e volume crítico podem ser observados na Tabela 4. Como dados experimentais para essas propriedades não foram encontrados na literatura, adotou-se o método de GANI para predizer essas propriedades do óleo da macaúba e os seus ésteres. Esse método foi escolhido, por ser o único dentre os estudados que necessita como entrada, apenas a estrutura molecular.

\subsection{Energia livre de Gibbs e Entalpia de Formação}

Os valores estimados da entalpia de formação e da energia livre de Gibbs, podem ser observados na Tabela 4.

Obteve-se na literatura dados da entalpia de formação do ácido esteárico, do éster metil oleato e etil oleato, respectivamente, $-884,7 \mathrm{~kJ} / \mathrm{mol},-734,5 \mathrm{~kJ} / \mathrm{mol}$ e $-775,8 \mathrm{~kJ} / \mathrm{mol}$ (NIST). Para esses compostos o método que apresentou menor desvio foi o de JOBACK. Portanto, este foi escolhido nos cálculos do óleo da macaúba e seus ésteres.

Para a energia livre de Gibbs o método escolhido foi o Benson (Reid et al.,1987). Segundo Aspen (2012), este é mais preciso na estimativa dessa propriedade que os métodos de GANI e JOBACK por considerar os efeitos dos átomos vizinhos nos cálculos.

\subsection{Propriedades para o Óleo da Polpa da Macaúba}

A caracterização do óleo da polpa da macaúba, assim como os seus respectivos ésteres metílicos e etílicos podem ser visualizados na Tabela 5.

Tabela 5 - Propriedades estimadas para o óleo da polpa da macaúba e ésteres

\begin{tabular}{ccccc}
\hline Propriedade & $\begin{array}{c}\text { Método de } \\
\text { contribuição } \\
\text { de grupos }\end{array}$ & $\begin{array}{c}\text { Óleo de } \\
\text { macaúba }\end{array}$ & $\begin{array}{c}\text { Ésteres } \\
\text { Metílicos }\end{array}$ & $\begin{array}{c}\text { Ésteres } \\
\text { Etílicos }\end{array}$ \\
\hline $\mathbf{T}_{\mathbf{b}}(\mathbf{K})$ & GANI & 622,20 & 598,30 & 608,10 \\
$\mathbf{T c}(\mathbf{K})$ & GANI & 793,45 & 769,00 & 777,83 \\
$\mathbf{P}_{\mathbf{C}}(\mathbf{b a r})$ & GANI & 12,76 & 11,38 & 10,75 \\
$\mathbf{V}_{\mathbf{C}}\left(\mathbf{m}^{\mathbf{3}} / \mathbf{k m o l}\right)$ & GANI & 1,031 & 1,090 & 1,146 \\
Entalpia de formação $(\mathbf{k J} / \mathbf{m o l})$ & JOBACK & $-658,19$ & $-645,33$ & $-666,04$ \\
Energia livre de Gibbs $(\mathbf{k J} / \mathbf{m o l})$ & BENSON & $-699,71$ & $-739,07$ & $-771,29$ \\
\hline
\end{tabular}

Goodrum (2002) determinou experimentalmente a temperatura de ebulição dos ésteres metílicos e etílicos obtidos dos óleos de soja, canola, colza e gordura animal. Para os ésteres metílicos observou 620,80 K (óleo de soja) a 642,14 K (óleo de colza), no entanto para os ésteres etílicos encontrou-se $628,39 \mathrm{~K}$ e $635,39 \mathrm{~K}$ (óleo de colza). Yuan et al. (2003) determinaram as propriedades críticas para os ésteres metílicos do óleo de soja, a temperatura, pressão e volume críticos obtidos, respectivamente, foi $785,7 \mathrm{~K}, 12,08$ bar e $1,082 \mathrm{~m}^{3} / \mathrm{kmol}$. Os dados encontrados neste trabalho encontram-se em faixas aceitáveis quando comparado com dados experimentais de óleos vegetais similares. O óleo da polpa da macaúba tem por composto principal o ácido oleico (67\%), enquanto que na soja o ácido linoleico é predominante (56\%). Essa diferença na composição dos óleos, principalmente no número de insaturações, provocam alterações nas propriedades termodinâmicas. 
Tabela 4 - Dados estimados de pressão crítica, volume crítico, entalpia de formação e energia livre de Gibbs dos ácidos graxos e ésteres.

\begin{tabular}{|c|c|c|c|c|c|c|c|c|c|c|c|c|c|c|c|}
\hline & & \multicolumn{4}{|c|}{$\mathbf{P}_{\mathrm{C}}$ (bar) } & \multicolumn{4}{|c|}{$V_{C}\left(\mathrm{~m}^{3} / \mathbf{k m o l}\right)$} & \multicolumn{3}{|c|}{ Entalpia (kJ/mol) } & \multicolumn{3}{|c|}{ Gibbs $(\mathrm{kJ} / \mathrm{mol})$} \\
\hline & & Joback & Gani & Lydersen & Ambrose & Joback & Gani & Lydersen & Ambrose & Joback & Gani & Benson & Joback & Gani & Benson \\
\hline \multirow{9}{*}{$\begin{array}{l}\text { Ácidos } \\
\text { graxos }\end{array}$} & C14:0 & 16,351 & 16,356 & 16,987 & 16,443 & 0,844 & 0,842 & 0,835 & 0,836 & $-682,56$ & $-680,51$ & $-415,76$ & $-276,91$ & $-273,53$ & $-644,12$ \\
\hline & C16:0 & 14,080 & 14,177 & 15,098 & 14,659 & 0,956 & 0,953 & 0,945 & 0,947 & $-723,84$ & $-722,04$ & $-455,62$ & $-260,07$ & $-257,06$ & $-708,45$ \\
\hline & C16:1 & 14,942 & 14,150 & 15,318 & 18,982 & 0,931 & 0,940 & 0,931 & 0,846 & $-614,70$ & $-609,10$ & $-387,75$ & $-170,02$ & $-179,93$ & $-610,99$ \\
\hline & C18:0 & 12,251 & 12,440 & 13,605 & 13,242 & 1,068 & 1,065 & 1,055 & 1,057 & $-765,12$ & $-763,56$ & $-495,48$ & $-243,23$ & $-240,60$ & $-772,77$ \\
\hline & C18:1 & 12,949 & 12,419 & 13,784 & 14,972 & 1,043 & 1,051 & 1,041 & 0,982 & $-655,98$ & $-650,63$ & $-441,43$ & $-153,18$ & $-163,46$ & $-716,19$ \\
\hline & C18:2 & 13,707 & 12,398 & 13,967 & 17,079 & 1,018 & 1,038 & 1,027 & 0,907 & $-546,84$ & $-537,69$ & $-359,74$ & $-63,13$ & $-86,33$ & $-577,87$ \\
\hline & C18:3 & 14,535 & 12,377 & 14,155 & 19,685 & 0,993 & 1,025 & 1,013 & 0,831 & $-437,70$ & $-424,75$ & $-291,87$ & 26,92 & $-9,19$ & $-480,41$ \\
\hline & C20:0 & 10,757 & 11,035 & 12,080 & 11,782 & 1,180 & 1,176 & 1,165 & 1,167 & $-806,40$ & $-805,09$ & $-535,34$ & $-226,39$ & $-224,14$ & $-837,10$ \\
\hline & C14:0 & 12,728 & 9,405 & 13,069 & 18,214 & 1,051 & 1,251 & 1,068 & 0,887 & $-425,35$ & $-450,29$ & $-346,63$ & 68,88 & 75,42 & $-548,01$ \\
\hline \multirow{8}{*}{$\begin{array}{c}\text { Ésteres } \\
\text { Metílicos }\end{array}$} & C16:0 & 12,355 & 12,546 & 13,865 & 13,789 & 1,014 & 1,012 & 1,000 & 1,002 & $-711,49$ & $-685,29$ & $-510,38$ & $-218,11$ & $-197,15$ & $-776,04$ \\
\hline & C16:1 & 13,061 & 12,525 & 14,052 & 15,682 & 0,989 & 0,999 & 0,986 & 0,927 & $-602,35$ & $-572,35$ & $-442,51$ & $-128,06$ & $-120,01$ & $-678,59$ \\
\hline & C18:0 & 10,842 & 11,121 & 12,597 & 12,529 & 1,126 & 1,124 & 1,110 & 1,112 & $-752,77$ & $-726,81$ & $-550,24$ & $-201,27$ & $-180,69$ & $-840,37$ \\
\hline & C18:1 & 11,421 & 11,103 & 12,750 & 14,074 & 1,101 & 1,110 & 1,096 & 1,037 & $-643,63$ & $-613,88$ & $-482,37$ & $-111,22$ & $-103,55$ & $-742,91$ \\
\hline & C18:2 & 12,048 & 11,086 & 12,908 & 15,938 & 1,076 & 1,097 & 1,082 & 0,962 & $-534,49$ & $-500,94$ & $-414,50$ & $-21,17$ & $-26,41$ & $-645,46$ \\
\hline & C18:3 & 12,728 & 11,069 & 13,069 & 18,214 & 1,051 & 1,084 & 1,068 & 0,887 & $-425,35$ & $-388,00$ & $-346,63$ & 68,88 & 50,72 & $-548,01$ \\
\hline & C20:0 & 9,591 & 9,952 & 11,560 & 11,499 & 1,238 & 1,235 & 1,220 & 1,222 & $-794,05$ & $-768,34$ & $-590,10$ & $-184,43$ & $-164,23$ & $-904,69$ \\
\hline & C14:0 & 13,233 & 13,379 & 14,623 & 14,542 & 0,958 & 0,956 & 0,945 & 0,947 & $-690,85$ & $-664,52$ & $-490,45$ & $-226,53$ & $-205,38$ & $-743,88$ \\
\hline \multirow{7}{*}{$\begin{array}{l}\text { Ésteres } \\
\text { Etílicos }\end{array}$} & C16:0 & 11,561 & 11,797 & 13,200 & 13,129 & 1,070 & 1,068 & 1,055 & 1,057 & $-732,13$ & $-706,05$ & $-530,31$ & $-209,69$ & $-188,92$ & $-808,20$ \\
\hline & C16:1 & 12,200 & 11,778 & 13,370 & 14,835 & 1,045 & 1,055 & 1,041 & 0,982 & $-622,99$ & $-593,11$ & $-462,44$ & $-119,64$ & $-111,78$ & $-710,75$ \\
\hline & C18:0 & 10,188 & 10,508 & 12,393 & 12,327 & 1,182 & 1,180 & 1,165 & 1,167 & $-773,41$ & $-747,58$ & $-570,17$ & $-192,85$ & $-172,46$ & $-872,53$ \\
\hline & C18:1 & 10,715 & 10,493 & 12,186 & 13,388 & 1,157 & 1,166 & 1,151 & 1,092 & $-664,27$ & $-634,64$ & $-502,30$ & $-102,80$ & $-95,32$ & $-775,08$ \\
\hline & C18:2 & 11,283 & 10,477 & 12,330 & 12,454 & 1,132 & 1,153 & 1,137 & 1,127 & $-555,13$ & $-521,70$ & $-434,43$ & $-12,75$ & $-18,18$ & $-677,62$ \\
\hline & C18:3 & 11,899 & 10,461 & 12,477 & 13,959 & 1,107 & 1,140 & 1,123 & 1,052 & $-445,99$ & $-408,76$ & $-366,56$ & 77,30 & 58,96 & $-580,17$ \\
\hline & C20:0 & 9,045 & 9,445 & 11,095 & 11,037 & 1,294 & 1,291 & 1,275 & 1,277 & $-814,69$ & $-789,10$ & $-610,03$ & $-176,01$ & $-155,99$ & $-936,85$ \\
\hline
\end{tabular}




\section{CONCLUSÕES}

Conclui-se que algumas propriedades de compostos podem ser estimadas satisfatoriamente por métodos de contribuição de grupo. A seleção do método é fator primordial na precisão do resultado final da estimativa. Neste trabalho, algumas propriedades foram estimadas com desvio satisfatório para aplicações em problemas de engenharia.

\section{REFERENCIAS}

ASPEN. (2012). Manual Aspen Plus v7.0.

BASSO, R. C.; SILVA, C. A. S.; SOUSA, C. O.; MEIRELlES, A. J. A.; BATISTA, E. A. C.(2013) LLE experimental data, thermodynamic modeling and sensitivityanalysis in the ethyl biodiesel from macauba pulp oil settling step. Bioresource Technology 131, 468-475.

CERIANI, R.; MEIRELLES, A. J. A. (2003) Predicting vapor-liquid equilibria of fatty systems. Fluid Phase Equilib., 215, 227-236.

CONSTANTIUNOU, L.; GANI, R..(1994) New group contribution method for estimating properties of pure compounds. AIChe Journal, 40 (10): p.1697-1710.

CORDES, W.; RAREY, J. (2002) A new method for the estimation of the normal boiling point of non-electrolyte organic compounds. Fluid Phase Equilib., 201, 409-433.

Dortmund Data Bank (DDB). Disponível em <www.ddbst.com>. Acesso em fev. de 2014.

DEMIRBAS, A. (2005) Biodiesel production from vegetable oils via catalytic and noncatalytic supercritical methanol transesterification methods. Progress In Energy and Combustion Science. no. 31. p. 466-487.

GOODRUM, J.W. (2002) Volatility and boiling points of biodiesel from vegetables oils and tallow. Biomass and Bioenergy. 22, p 205-211.

JOBACK, K.G. e REID, R.C. (1987) Estimation of pure-component properties from group contributions. Chem. Eng. Communications, 57:1-6, 233-243.

LOPES, D. C.; NETO, A. J. S.; MENDES, A. A.; PEREIRA, D. T. V.(2013) Economic feasibility of biodiesel production from Macauba in Brazil. E. Economics 40, 819-824.

NIST. Disponível em: <http://www.nist.gov/>. Acesso em fev. 2014.

PIMENTA, T.V.2010. Metodologias de obtenção e caracterização dos óleos do fruto da macaúba com qualidade alimentícia: da coleta à utilização. MSc Eng. química, UFMG.

REID, R. C., J. M. PRAUSNITZ, AND T. K. SHERWOOD. (1987). The Properties of Gases and Liquids. 4th ed. New York, N.Y.: McGraw-Hill.

REZENDE, D. B. (2011) Esterificação e transesterificação de óleos de macaúba com elevada e baixa acidez catalisadas por resinas de troca iônic $a$. MSc Eng. Química., UFMG.

ZEBERG, C. K.; STENBY, E. H. (1999) Predicting the melting points and the enthalpies of fusion of saturated triglycerides by a group contribution method. Fluid Phase Eq., 162, 7-17.

YUAN, W.; HANSEN, A. C.; ZHANG, Q. (2003) Predicting the physical properties of biodiesel for combustion modeling. Transactions of ASAE. 46(6), p 1487-1493

\section{Agradecimentos a FAPEMIG}

